This item was submitted to Loughborough's Research Repository by the author.

Items in Figshare are protected by copyright, with all rights reserved, unless otherwise indicated.

\title{
Properties and application of polyimide-based composites by blending surface functionalized boron nitride nanoplates
}

\section{PLEASE CITE THE PUBLISHED VERSION}

http://dx.doi.org/10.1002/app.41889

\section{PUBLISHER}

(C) Wiley-Blackwell

\section{VERSION}

AM (Accepted Manuscript)

\section{PUBLISHER STATEMENT}

This work is made available according to the conditions of the Creative Commons Attribution-NonCommercialNoDerivatives 4.0 International (CC BY-NC-ND 4.0) licence. Full details of this licence are available at: https://creativecommons.org/licenses/by-nc-nd/4.0/

\section{LICENCE}

CC BY-NC-ND 4.0

\section{REPOSITORY RECORD}

Chen, Yuanming, Xing Gao, Jinling Wang, Wei He, Vadim V. Silberschmidt, Shouxu Wang, Zhihua Tao, and Huan Xu. 2019. "Properties and Application of Polyimide-based Composites by Blending Surface Functionalized Boron Nitride Nanoplates”. figshare. https://hdl.handle.net/2134/16974. 


\title{
Properties and application of polyimide-based composites by blending surface functionalized boron nitride nanoplates
}

\author{
Yuanming Chen, ${ }^{1,2}$ Xing Gao, ${ }^{2}$ Jinling Wang, ${ }^{2}$ Wei He, ${ }^{1}$ Vadim V. Silberschmidt, ${ }^{2}$ Shouxu Wang, ${ }^{2}$ \\ Zhihua Tao, ${ }^{2}$ Huan $\mathrm{Xu}^{3}$ \\ ${ }^{1}$ State Key Laboratory of Electronic Thin Films and Integrated Devices, University of Electronic Science and Technology of China, \\ Chengdu 610054, People's Republic of China \\ ${ }^{2}$ Wolfson School of Mechanical and Manufacturing Engineering, Loughborough University, Loughborough LE11 3TU, United \\ Kingdom \\ ${ }^{3}$ Research and Development Department, Bomin Electronic Co., Ltd, Meizhou 514000, China \\ Correspondence to: W. He (E-mail: heweiz@uestc.edu.cn)
}

ABSTRACT: Ball milling was used to decrease the particle size of boron nitride (BN) nanoplates and to form more silylated functionalization of their surface. Such functionalized BN nanoplates enhanced their homogeneous dispersion in polyimide (PI) matrix. Thermal conductivities, thermal stabilities, and dielectric properties were characterized to investigate the particular effects on the performance of PI-based composites with functionalized BN nanoplates. When the concentration of functionalized BN nanoplates increased to $50 \mathrm{wt} \%$, thermal conductivity of the composite increased up to $1.583 \mathrm{~W} \mathrm{~m} \mathrm{~m}^{21} \mathrm{~K}^{21}$, and the temperature of final thermal decomposition was improved to $600^{\circ} \mathrm{C}$. An increasing trend was found in the dielectric constant of the composites while the dielectric loss decreased with the increase in the fraction. An appropriate fraction of functionalized BN nanoplates in PI-based composites were necessary to meet the requirement of withstanding drilling forces for the interconnecting through holes of flexible circuits. $\checkmark 2015$ Wiley Periodicals, Inc. J. Appl. Polym. Sci. 2015, 132, 41889.

KEYWORDS: applications; composites; properties and characterization; thermal properties

Received 1 August 2014; accepted 14 December 2014

DOI: 10.1002/app.41889

\section{INTRODUCTION}

Three-dimensional (3D) interconnections with dynamic or static flexible circuits have played an important part in electronic packaging since flexible circuits can be employed in flexible electronics to reduce package size and weight, decrease assembly time and cost, increase system reliability and replace point-to-point wires. ${ }^{1,2}$ However, as the electronic industry moves toward the design of ultra large-scale integrated circuits, heat conduction in interconnects of flexible circuits with polymeric substrate has challenged the performance and reliability of microelectronic and optoelectronic devices. ${ }^{3,4}$ Heat accumulated in flexible circuits often induces thermal fatigue and chemical reactions, leading to low service life and operation efficiency of electronic systems. ${ }^{5}$

Amorphous polymer materials for the substrates of flexible circuits are generally regarded as thermal insulators because of their low-thermal conductivity. ${ }^{6}$ No electrons for free movement exist in these amorphous polymers so that phonons, quantized modes of vibration occurring in a rigid crystal lattice are the major heat carriers for heat conduction in most polymers. $^{7-10}$ Low-thermal conductivity of polymer materials is caused by slow heat transport of phonons along the tempera-

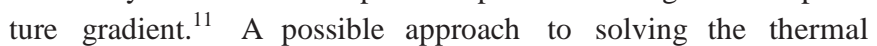
problem of devices based on polymeric flexible circuits is to enhance thermal conductivity of polymers with highly conductive inorganic fillers. Different inorganic fillers have been used to improve performance (i.e., thermal conductivity) of polymer matrix since they can form more continuous heat-conducting chains in the matrix. ${ }^{7,10-15}$ Boron nitride $(\mathrm{BN})$ is frequently used as an insulating thermoconductive filler in polymer-based composites. $^{16} \mathrm{Li}$ et al. ${ }^{17}$ employed granular micro-BN to improve thermal conductivity of novolac resin. The mixture of BN particles sized $15 \mu \mathrm{m}$ and $0.5 \mu \mathrm{m}$ with a mass ratio of 1 : 2 provided BN/novolac resin composites with maximum thermal conductivity. $\mathrm{Ng}$ et al. ${ }^{18}$ added $\mathrm{BN}$ particles with different particle sizes into thermoplastics to enhance thermal networks of composite matrix. BN-filled thermoplastics exhibited higher value of thermal conductivity at a higher concentration of $\mathrm{BN}$ filler. 
Polyimide (PI) is one of polymer materials with low-thermal conductivity widely applied as a dielectric material of flexible circuits due to its excellent thermal stability, mechanical properties, and a low dielectric constant. ${ }^{19,20}$ According to early works, PI was modified with inorganic fillers to enhance its thermal conductivity. Wang et al. ${ }^{21}$ pointed out that thermal conductivity of aluminum nitride (AlN)/PI composites could significantly increase when the volume fraction of AlN reached $60 \%$. However, an unexpected reaction between AlN particles and water could be induced when AlN/PI composite systems served in wet environment. ${ }^{22} \mathrm{BN}$ particles have been also used to enhance thermal conductivity of PI to achieve stable performance of polymeric composites. Li et al. ${ }^{23}$ found that $30 \mathrm{wt} \%$ of microand nanosized $\mathrm{BN}$ at the weight ratio of $7: 3$ in BN/PI composite matrix was sufficient to form largest thermally conductive path to dissipate the heat. However, the shape of the dispersed particles could also play a role in the determination of the effective thermal conductivity of particle-filled polymers. ${ }^{21,24}$ Nanoplate fillers with high aspect ratios easily form two-dimensional conductive networks for phonon transport and provide heat dissipation in the in-plane direction of composites. ${ }^{6,25-27}$ Nanoplate fillers could also provide good dispersion and enhance conductivity properties of polymer materials. ${ }^{25}$

In this work, functionalized $\mathrm{BN}$ nanoplates were employed to enhance thermal conductivities of PI-based composites. A ballmilling process was used to further decrease the size of $\mathrm{BN}$ nanoplates and form maximized silylation on their surface. Thermal and dielectric properties of developed PI-based composite systems were tested to investigate the effects of functionalized BN nanoplates on composite systems. Functionalized BN nanoplates/PI composites were also drilled to obtain through holes for interconnects of flexible circuits after metallization.

\section{EXPERIMENTAL}

Unfunctionalized BN nanoplates with hexagonal structure and a purity of $>98.2 \%$ were supplied by Shandong Pengcheng Special Ceramics Ltd., China. $4 \mathrm{~g}$ of BN nanoplate powder was injected in a mixed solution with $90 \mathrm{~mL}$ methyltriethoxysilane, $280 \mathrm{~mL}$ ethanol, and $30 \mathrm{~mL}$ water and glacial acetic acid to regulate $\mathrm{pH}$ value of 3 . The mixture was ball-milled by agate balls for $24 \mathrm{~h}$ in a planetary ball mill and then centrifuged at $2000 \mathrm{rpm}$ for $15 \mathrm{~min}$. The precipitate was subsequently dried at $120^{\circ} \mathrm{C}$ for $12 \mathrm{~h}$ under vacuum to completely remove the solvent. Thereafter, as-received functionalized BN were grinded in an agate mortar and sieved (mesh size $5 \mathbf{~ I m}$ ) to facilitate homogeneous nanoplates.

At the next stage, $0.745 \mathrm{~g}$ 1,4-diaminobenzene was injected into a 50-mL three-neck flask containing $20 \mathrm{~mL} \mathrm{~N}, \mathrm{~N}$-dimethylformamide solution under a mechanical stirrer. About $1.504 \mathrm{~g}$ pyromellitic dianhydride was then added, when 1,4-diaminobenzene was completely dissolved. Above mixture was stirred in an ice bath $\left(0-5^{\circ} \mathrm{C}\right)$ for $1 \mathrm{~h}$ to obtain a viscous solution. Thereafter, functionalized $\mathrm{BN}$ nanoplates at concentrations of $5 \mathrm{wt} \%, 10$ wt \%, 20 wt \%, 30 wt \%, 40 wt \%, and 50 wt \% were, respectively, injected into one viscous solution with vigorous stirring for $10 \mathrm{~h}$ at $20^{\circ} \mathrm{C}$ to allow for completing the reduction reaction and avoiding local aggregation. The mixture was filtered and casted onto clean glass plates, subsequently dried in a vacuum oven at $80^{\circ} \mathrm{C}$ for $5 \mathrm{~h}$ to obtain polyamic acid after volatilization of DMF solvent. As-received film was further heated at $310^{\circ} \mathrm{C}$ for $12 \mathrm{~h}$ in a heating oven to convert polyamic acid film to PI film blended with functionalized BN nanoplates. Thus, PI/functionalized BN nanoplates composite with low price was finally obtained.

Microstructures of BN nanoplates and composites were observed by a field emission scanning electron microscope (FESEM) (HITACHI S3400) coupled to an energy-dispersive Xray spectrometer (EDS). A laser granulometer (CHENGDU JINGXIN JL1197) was employed to evaluate the particle size distributions of BN nanoplates and functionalized BN nanoplates. Fourier-transform infrared (FTIR) spectroscope (SHIMADZU FTIR8000) allowed for the determination of the chemical composition of BN nanoplates and composites. Surfaces of pure PI and composites were studied with a metallographic microscope (ASIDA JX23). A laser-flash thermal conductive measurement (NETZSCH LFA-467) was used to test the thermal conductivity of PI and composites, while the assessment of thermal stability was performed with a simultaneous thermogravimetry/differential thermal analyzer (METTLER TOLEDO TGA/DSC-1) in a range from $50^{\circ} \mathrm{C}$ to $600^{\circ} \mathrm{C}$ at a heating rate of $10^{\circ} \mathrm{C} \min ^{21}$ under air atmosphere. Measurements of dielectric properties were performed for the frequency ranging from 0 to $30 \mathrm{MHz}$ on a broadband dielectric spectrometer (AGILENT 4285A) with a high-performance frequency analyzer. The composite specimens with dimensions of $5 \mathrm{mmx}$ $5 \mathrm{~mm}$ and thickness of $18 \mu \mathrm{m}$ for these measurements were double-sided laminated by 12.5 - $\mu$ m-thick copper foils. Functionalized BN nanoplates/PI composites were drilled with a computer numerical control (CNC) drilling machine (HITACHI MARK-20D) to investigate the effect of $\mathrm{BN}$ particles on their machinability. Through holes in the functionalized BN nanoplates/PI composites were electroplated to generate interconnects of flexible circuits.

\section{RESULTS AND DISCUSSION}

Surface Functionalized Mechanism of BN Nanoplates for Composites

Most inorganic fillers are immiscible in most organic polymer materials due to poor specific interactions within such organic/ inorganic composite systems and negligibly low combined entropy contribution to the free energy of mixing. ${ }^{28,29}$ Silane coupling agents can function at the interface of inorganic fillers to create a chemical bridge between the reinforcement and organic polymer. ${ }^{25,30}$ It means that such silane functionalization for absorbing silane molecules at the surface of inorganic fillers can improve wettability and dispersion of inorganic fillers in the polymer matrix. ${ }^{13,25,27}$ However, inorganic fillers are generally injected into a solution mixed with a silane coupling agent, alcohol, and water in the way of stir or agitation for silylated reaction ${ }^{17,23}$ while inorganic particles can immediately precipitate to form particle agglomerations. ${ }^{13}$ Limited silylation is absorbed at the surface of inorganic particles without their effective dispersion in the mixed solution. Ball milling can led 

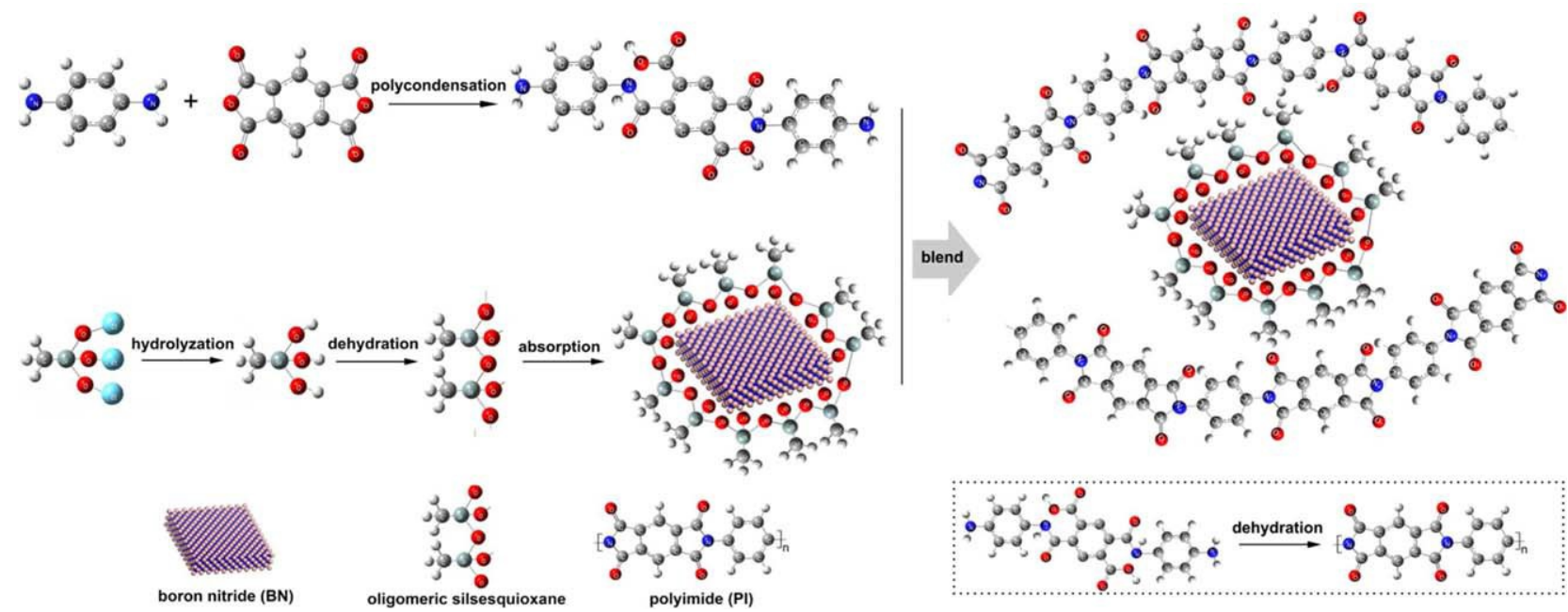

Figure 1. Schematic diagram of the preparation of functionalization of BN nanoplates and functionalized BN nanoplates/PI composites. [Color figure can be viewed in the online issue, which is available at wileyonlinelibrary.com.]

to a good result with the maximal dispersion of inorganic par-
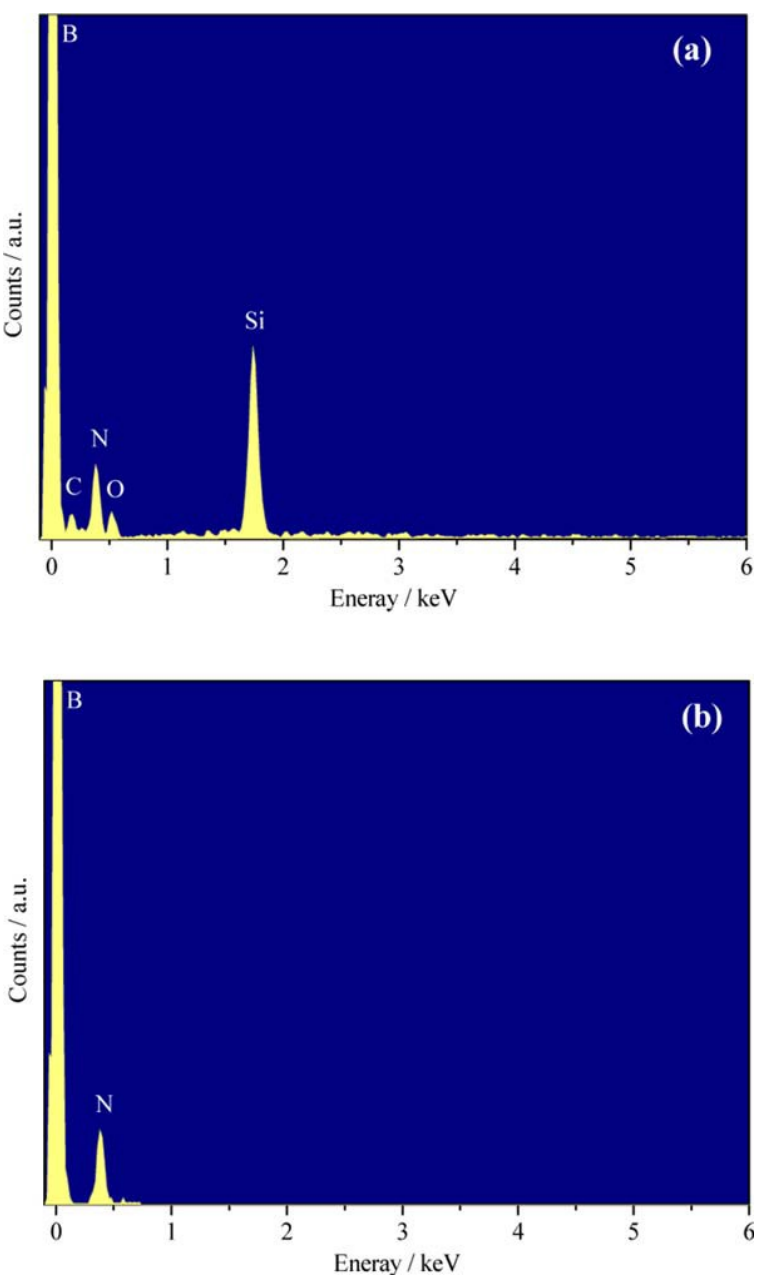

Figure 2. EDS spectra of functionalized BN nanoplates: functionalization of BN nanoplates (a); unfunctionalization of BN nanoplates (b). [Color figure can be viewed in the online issue, which is available at wileyonlinelibrary.com.] ticles for their covering with silane coupling agents due to mechanical rotation and the friction between inorganic particles and agate balls. During ball milling, methyltriethoxysilane successively underwent hydrolyzation, dehydration, and absorption at the surface of BN nanoplates, as shown in Figure 1. EDS analysis and FTIR spectra allowed for the determination of the silylation of $\mathrm{BN}$ nanoplates. Figure 2 of EDS spectra displays that the product was composed of $\mathrm{B}, \mathrm{N}, \mathrm{Si}, \mathrm{C}$, and $\mathrm{O}$ in functionalized $\mathrm{BN}$ nanoplates, compared to $\mathrm{B}$ and $\mathrm{N}$ in unfunctionalized ones. In addition, FTIR spectra of functionalized BN nanoplates (curve b of Figure 3) presented different characteristic absorption bands, compared to that of unfunctionalized BN nanoplates (curve a of Figure 3). BN nanoplates exhibited two characteristic vibrational modes as the in-plane axial B-N-B vibration, centered at about $1406 \mathrm{~cm}^{21}$ and the out-of-plane

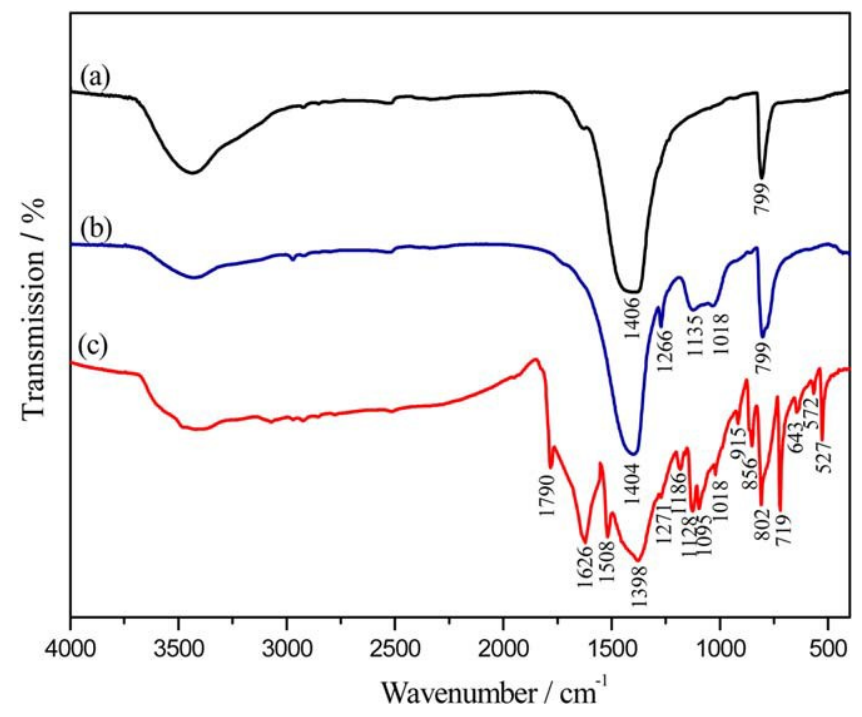

Figure 3. FTIR spectra of unfunctionalized BN nanoplates (curve a), functionalized BN nanoplates (curve b) and functionalized BN nanoplates/PI composite (curve c). [Color figure can be viewed in the online issue, which is available at wileyonlinelibrary.com.] 

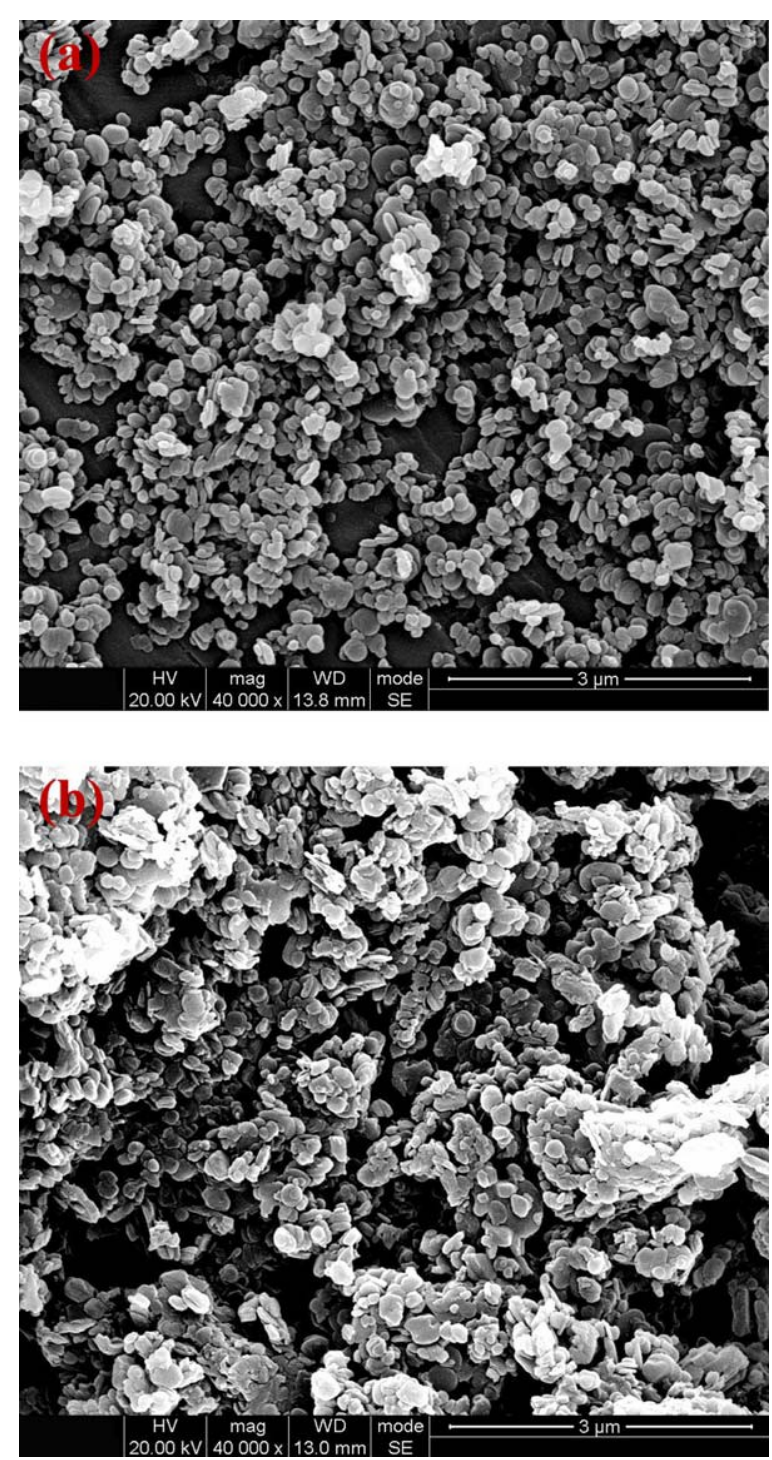

Figure 4. SEM micrographs of BN nanoplates: functionalized BN nanoplates (a); unfunctionalized BN nanoplates (b). [Color figure can be viewed in the online issue, which is available at wileyonlinelibrary.com.]

B-N vibration, centered at about $799 \mathrm{~cm}^{21}$. 31 The superimposed absorption at $1266 \mathrm{~cm}^{21}$ was due to the presence of $\mathrm{Si}-\mathrm{C}$ on the surface of silylated BN nanoplates. ${ }^{28}$ The absorption ranging from $1018 \mathrm{~cm}^{21}$ to $1135 \mathrm{~cm}^{21}$ corresponded to the stretching vibrations of siloxane $(\mathrm{Si}-\mathrm{O}-\mathrm{Si}){ }^{29}$ Thus, BN nanoplate was adsorbed by the oligomeric silsesquioxane, $\left(\mathrm{CH}_{3} \mathrm{SiO}_{1.5}\right)_{n}$, to generate functionalized groups for blending with PI.

From scanning electron microscope (SEM) micrographs of Figure 4, silylation-functionalized $\mathrm{BN}$ nanoplates were more dispersed than unfunctionalized ones. Early works indicated that the smallest filler particle size would give the highest specific surface energy to result in low-level uniform particle dispersion, 10,32 thereby causing aggregated BN nanoplates [Figure 4(b)]. However, as illustrated in Figure 4(a), functionalized BN nanoplates with surface reinforcement with oligomeric silsesquioxane could match the energy required to overcome the van der Waals forces. ${ }^{16}$ In other words, the formation of agglomeration could be inhibited, attributed to the decrease in surface energy between $\mathrm{BN}$ nanoplates with oligomeric silsesquioxane.

Ball milling for functionalized reaction could further reduce the mean particle sizes of BN nanoplates, as shown in Figure 5. The volume fraction of $\mathrm{BN}$ nanoplates ranging from $0.24 \mu \mathrm{m}$ to $0.38 \mu \mathrm{m}$ in the mean particle size increased to $40.80 \%$ after ball milling, compared to $28.75 \%$ before ball milling. Volume fractions of functionalized $\mathrm{BN}$ nanoplates with mean particle size of $0.34 \mu \mathrm{m}, 0.38 \mu \mathrm{m}, 0.41 \mu \mathrm{m}, 0.45 \mu \mathrm{m}$, and $0.49 \mu \mathrm{m}$ were $12.65 \%, 19.09 \%, 21.44 \%, 18.07 \%$, and $11.48 \%$, respectively. Functionalized BN nanoplates toward smaller sizes exhibited a significant increase of $2.65 \%, 4.08 \%, 3.76 \%$, and $0.97 \%$ in volume fraction for mean particle size of $0.32 \mu \mathrm{m}, 0.34 \mu \mathrm{m}, 0.38$ $\mu \mathrm{m}$, and $0.41 \mu \mathrm{m}$, respectively. The remarkable decrease in particle size of $\mathrm{BN}$ nanoplates resulted from mechanical grinding between agate balls with hardness of 7 (hardness of BN nanoplates during silylation was 2-4). Thus, smaller BN nanoplates could lead to a high surface area and induce more unsaturated chemical bonds at the surface ${ }^{10}$ so that more oligomeric silsesquioxane could be absorbed at the surface of $\mathrm{BN}$ nanoplates,
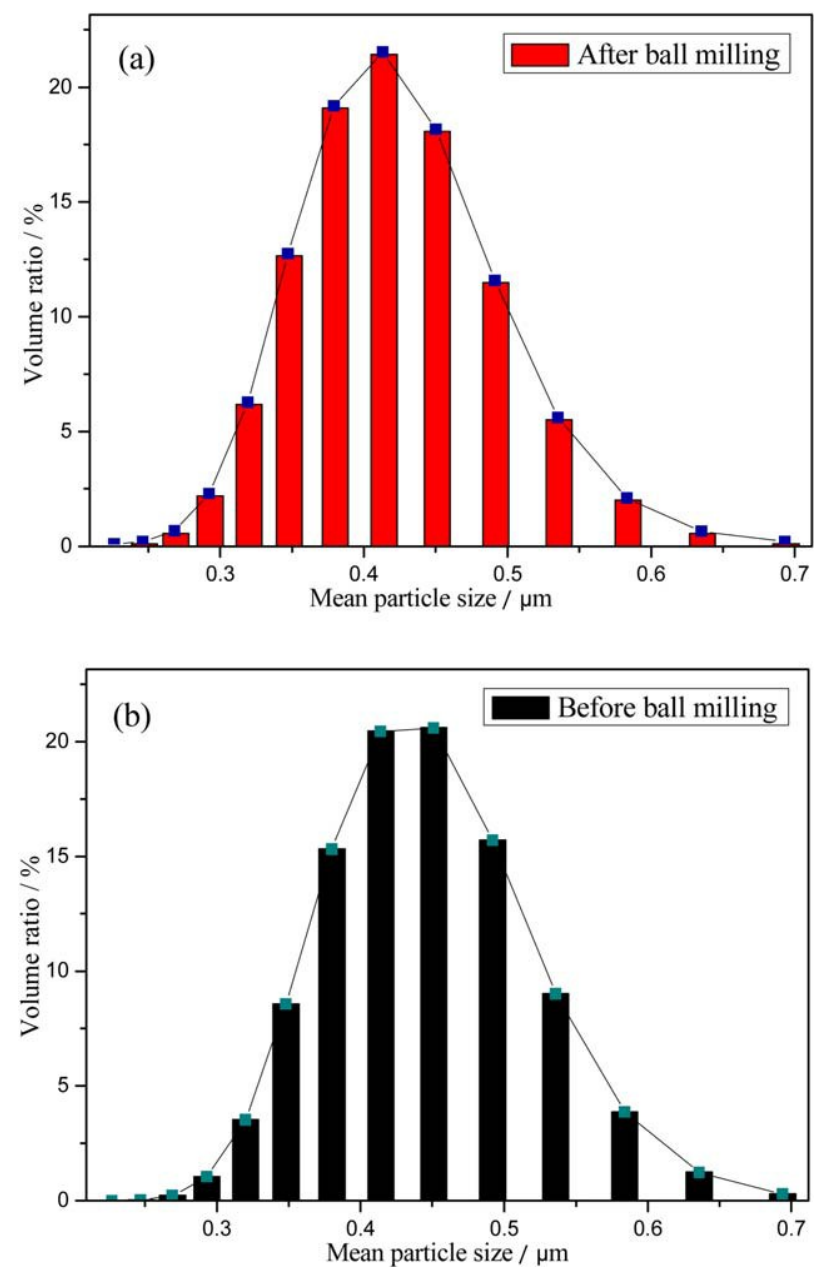

Figure 5. Particle size distributions of functionalized BN nanoplates (a), compared to unfunctionalized ones (b). [Color figure can be viewed in the online issue, which is available at wileyonlinelibrary.com.] 

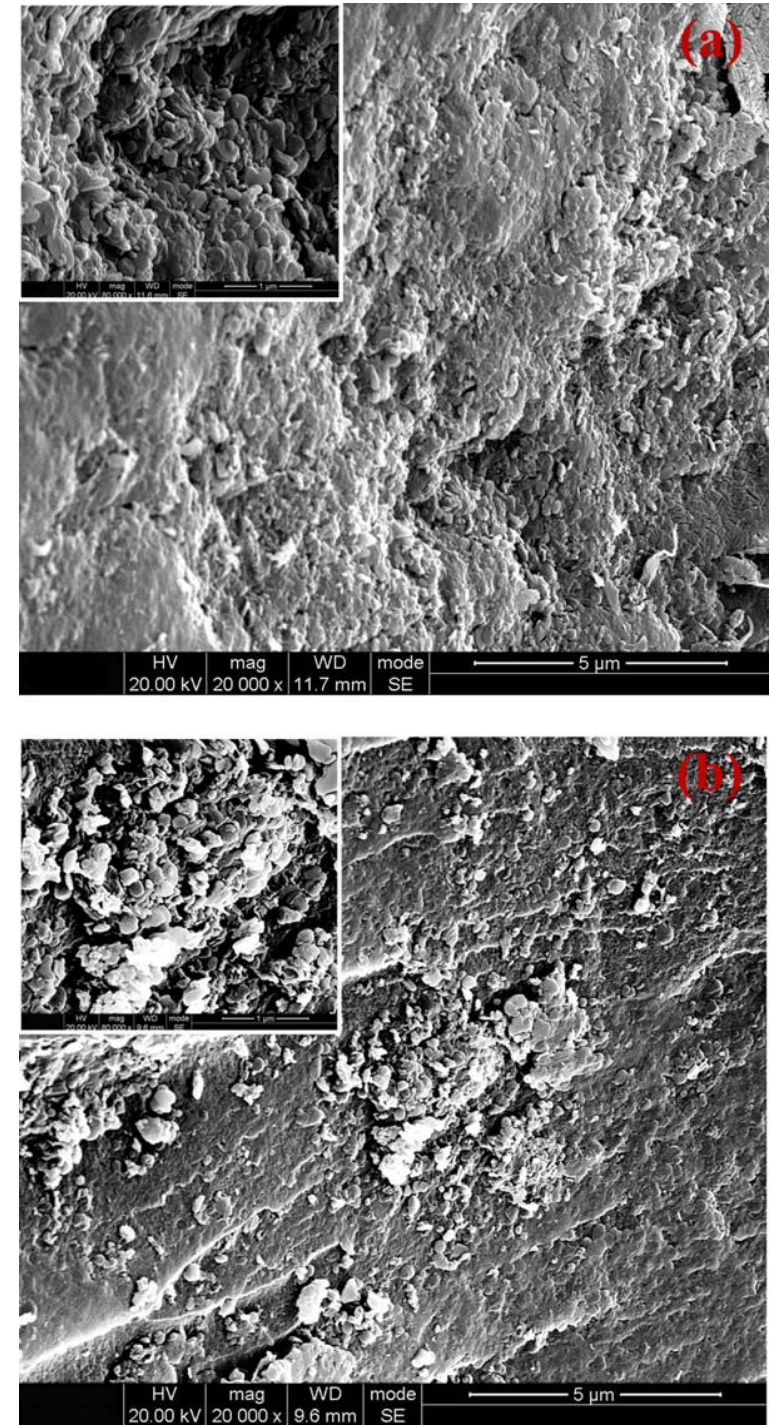

Figure 6. SEM micrographs of cross sections of PI-based composites blending with functionalized BN nanoplates (a) and unfunctionalized BN nanoplates (b). [Color figure can be viewed in the online issue, which is available at wileyonlinelibrary.com.]

thereby improving dispersion of functionalized $\mathrm{BN}$ nanoplates as well.

Functionalized BN nanoplates were blended into polyamic acid to form composites but PI-based composites could be only prepared after the dehydration of polyamic acid, as indicated in Figure 1. FTIR spectra of functionalized BN nanoplates/PI composite (curve c of Figure 3) displayed that the absorption at $1790 \mathrm{~cm}^{-1}$ attached to the asymmetrical and symmetrical stretching of $\mathrm{C}=\mathrm{O} .^{33}$ The absorption peaks at $1626 \mathrm{~cm}^{-1}$ and $1508 \mathrm{~cm}^{21}$ were assigned to the benzene ring skeleton vibration peak, while the absorptions at $915 \mathrm{~cm}^{-1}, 856 \mathrm{~cm}^{-1}, 643 \mathrm{~cm}^{-1}$, and $527 \mathrm{~cm}^{-1}$ corresponded to the phenyl characteristic absorption. Absorptions at $1398 \mathrm{~cm}^{-1}$ and $719 \mathrm{~cm}^{-1}$ were assigned to the stretching vibration peak and the flexible vibration summit of $\mathrm{C}-\mathrm{N}-\mathrm{C}$, respectively, and the absorption at $1128 \mathrm{~cm}^{-1}$ was due to the transverse imide of $\mathrm{C}-\mathrm{N}-\mathrm{C}$. The absorption peak at
$1018 \mathrm{~cm}^{21}$ was attributed to out-of-plane bending absorption of the amino groups. Above absorption peaks showed that polyamic acid reacted completely to produce PI after heating reaction.

Microstructures of PI-based composite with BN nanoplates were also observed to investigate the interfacial bonding of $\mathrm{BN}$ nanoplates surrounding or covering the polymeric matrix. Figure 6 presents the morphological structures of PI-based composites blended with functionalized $\mathrm{BN}$ nanoplates and unfunctionalized BN nanoplates. The latter induced the formation of local aggregation and were separated from the continuous phase of polymeric matrix. However, functionalized BN nanoplates exhibited better particle dispersion in the matrix due to the presence of oligomeric silsesquioxane at their surface. On the other hand, oligomeric silsesquioxane improved the interfacial adhesion when one end of the molecule (-Si-O-Si-) was tethered to the surface of reinforcing $\mathrm{BN}$ nanoplates and $-\mathrm{CH}_{3}$ at the other end combined with the polymeric phase in a noncovalent way. ${ }^{25,30}$ In addition, functionalized BN nanoplates with smaller particle sizes could also provide larger surface area for higher absorption energy to contact with polymer, signaling better developed particle dispersion in polymeric composite. ${ }^{34}$ Therefore, functionalized BN nanoplates could be well dispersed in PI matrix [Figure 6(a)].

Particular Effects on the Properties of Functionalized BN Nanoplates/PI Composites

Polymers could exhibit different thermal, dielectric, and mechanical behaviors when blended with inorganic fillers. As shown in Figure 7, thermal conductivity values of BNnanoplates-filled PI composites increased with the growth in the fractions of $\mathrm{BN}$ nanoplates. The character of relationship between thermally conductive values and the loadings of filler in composites could be divided into two main stages. The first stage exhibited a slow increase in thermal conductivity for the weight fractions ranging from $5 \mathrm{wt} \%$ to $20 \mathrm{wt} \%$ followed by a significantly increasing trend in the second stage. At concentrations from $30 \mathrm{wt} \%$ to $50 \mathrm{wt} \%$, thermal conductivity of PIbased composite systems jumped to large values, indicating that it was determined mainly by the heat transport of the highly heat conductive BN nanoplates. ${ }^{13}$ However, PI-based composite with silylation-functionalized $\mathrm{BN}$ nanoplates exhibited higher values of thermal conductivity than that with unfunctionalized BN nanoplates. It was observed that the addition of functionalized BN nanoplates to the PI matrix resulted in a lower increase in thermal conductivity than that for unfunctionalized $\mathrm{BN}$ nanoplates when their fraction was lower than $20 \mathrm{wt} \%$. Thermal conductive value was higher only by $0.10 \mathrm{~W} \mathrm{~m}^{-1} \mathrm{~K}^{-1}$ in the PI-based composite with functionalized $\mathrm{BN}$ nanoplates, compared to that with unfunctionalized $\mathrm{BN}$ nanoplates at the same fraction of $20 \mathrm{wt} \%$. This value reached a threshold value when the fraction of $\mathrm{BN}$ nanoplates increased up to $30 \mathrm{wt} \%$. Functionalized BN nanoplates further improved thermal conductivity of the PI-based composite when more BN nanoplates were added. Thermal conductivity grew 7-fold (compared to pure PI) for the PI-based composite containing $50 \mathrm{wt} \%$ fillers from 0.221 to $1.583 \mathrm{~W} \mathrm{~m}^{-1} \mathrm{~K}^{-1}$. Such a value of thermal conductivity was higher by $0.26 \mathrm{~W} \mathrm{~m}^{-1} \mathrm{~K}^{-1}$ than that in the 

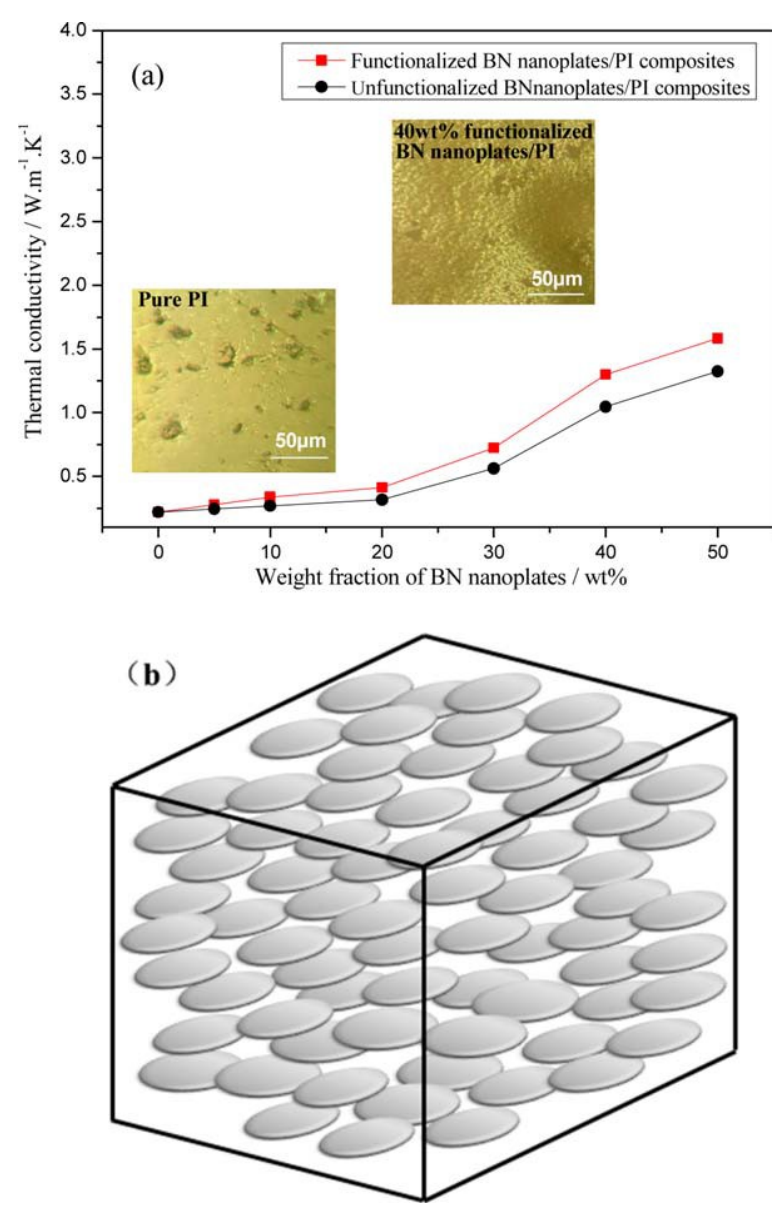

Figure 7. Thermal conductivities of filled BN nanoplates/PI blend as a function of BN nanoplate concentration (a) and thermal conductive networks of BN nanoplates in PI matrix (b): inserts of (a) displayed the surficial morphologies of pure PI and composite. [Color figure can be viewed in the online issue, which is available at wileyonlinelibrary.com.]

composite with unfunctionalized BN nanoplates. Addition of functionalized BN nanoplates induced lager increases in thermal conductivity of polymeric matrix.

Thermal conductivity of the polymer-based composites with inorganic filler could be influenced by filler loadings, surface roughness, particle size, and size distribution as well as surface treatment. ${ }^{6,735}$ Remarkable increases in thermal conductivity were found in the PI-based composites when adding more BN nanoplates since BN nanoplates with a high aspect-ratio structure could easily form more two-dimensional conductive channels for phonon transport, as shown in Figure 7(b). In other words, the rapid growth of thermal conductivity could be attributed to the significant conductive pathways of highly conductive $\mathrm{BN}$ nanoplates in the composite system, while the distance between $\mathrm{BN}$ nanoplates reduced as their concentration increased. In addition, materials with roughened surfaces exhibited lower thermal conductivity. ${ }^{36,37}$ Fewer voids in the composites would be generated on the surface and in interior of the composite system with an increase in the filler concentration due to stronger interfacial interaction between the filler with silylated functionalization and polymeric matrix. ${ }^{38,39}$ Thus, surface morphology of functionalized BN nanoplates/PI composite exhibited fewer voids after solvent volatilization of curing process, compared to that for pure PI, illustrated in the inserts of Figure 7(a). Addition of BN nanoplates could enhance the chance of solvent volatilization in the way of solvent penetration between particles and polymer. Thus, fewer voids for decreasing the scattering of phonons could improve the contact of $\mathrm{BN}$ nanoplates in the composite to form conductive networks, thereby strengthening phonon transport and increasing thermal conductivity. ${ }^{17,18}$ Furthermore, the decrease in particle size and the good dispersion of the filler in the composite systems could play a significant role in the determination of the effective thermal conductivity. ${ }^{40}$

Ball milling mechanically forced the distribution of BN nanoplates toward smaller sizes so that more small BN nanoplates could form conductive links for large ones, causing a synergistic effect between small and large BN nanoplates for heat dissipation of phonons. ${ }^{41}$ For a two-phase composite system with inorganic filler and polymer, the interfacial physical contact between polymer and filler became critical due to the sensitivity of phonons to interfacial defects, ${ }^{42}$ inducing the acoustic impedance mismatch at the interface between the fillers and the polymeric matrix. ${ }^{13}$ An interfacial resistance between the fillers and the surrounding matrix termed the resistance posed an interfacial thermal barrier to heat flow that might inhibit the benefit of adding highly conductive filler. ${ }^{10,43,44}$ In the consolidation of the composite, voids or air pockets would exist in large agglomerates of unfunctionalized $\mathrm{BN}$ nanoplates in the PI matrix [Figure 6(b)], leading to lower thermal conductivity of the composite. ${ }^{27}$ However, acoustic phonon mismatch and weak bonding between the PI matrix and unfunctionalized BN nanoplates could be addressed by introducing a better developed interfacial bonding, which could be engineered by a silylated functionalization process. On the other hand, oligomeric silsesquioxane from the silylation process could bridge well-dispersed BN nanoplates in the PI matrix [Figure 6(a)] and decrease the resistance to the heat flow caused by the compact interface between PI matrix and functionalized BN nanoplates. ${ }^{27}$ Therefore, silylated functionalization of BN nanoplates with a highaspect-ratio structure and small-sized particles for good dispersion in polymeric matrix contributed to higher thermal conductivity of the PI-based composites containing functionalized BN nanoplates than that for a case of unfunctionalized ones.

A heat-load increase could be employed to investigate the thermal stability of polymeric materials. Thermogravimetry (TG) analysis of composites with different concentrations of functionalized BN nanoplates illustrated the effects of functionalized BN nanoplates on thermal decomposition with two weight-loss stages, as indicated in Figure 8. Pure PI exhibited high thermooxidative stability due to the existence of benzene rings and imide rings. However, the thermo-oxidative decomposition behaviors of functionalized BN nanoplates/PI composites demonstrated similar characteristics to that of pure PI. However, initial decomposition temperatures of functionalized BN nanoplates/PI composites were higher than that of pure PI, as shown in the inserting DSC curves of Figure 8 since functionalized BN nanoplates as the obstacle with high fractions could not only affect the copolymerization of long network chain of polymer 


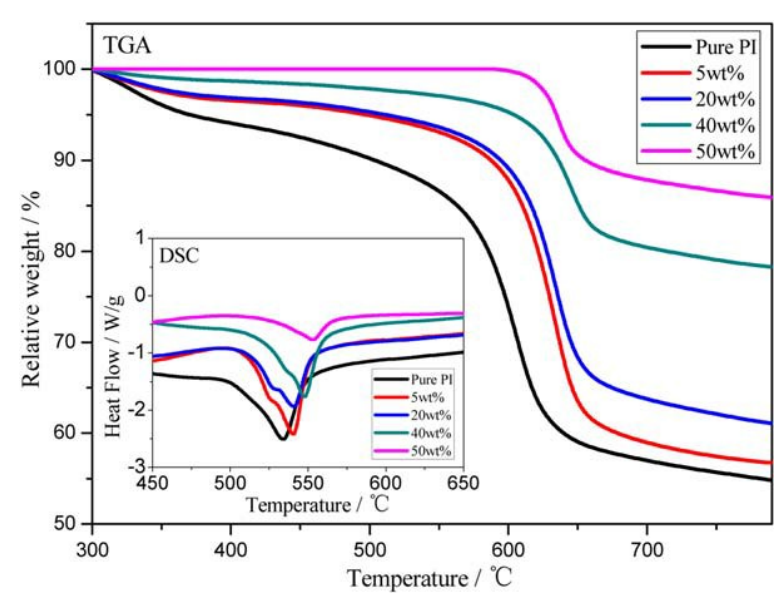

Figure 8. Thermogravimetric profiles (TGA) and DSC curves of pure PI and PI-based composites containing different fractions by weight of functionalized $\mathrm{BN}$ nanoplates. [Color figure can be viewed in the online issue, which is available at wileyonlinelibrary.com.]

but also absorb heat to reduce the thermal effects of polymer because of their high heat resistance. ${ }^{17}$ A continuous weight reduction in decomposition of the oligomer was observed for functionalized $\mathrm{BN}$ nanoplates/PI composites compared to the drastic weight loss of pure PI since highly thermal conductive BN nanoplates enhanced both effects of heat transmission and heat absorption. However, the thermal effects of functionalized $\mathrm{BN}$ nanoplates in composites could not influence the decomposition temperature of the polymer at $550^{\circ} \mathrm{C}$ due to the thermal stability of PI. Thermal decomposition of the composite system with low concentration of functionalized $\mathrm{BN}$ nanoplates was much more rapid than that for high concentration and the decomposition rate of composites decreased with the increasing fraction of functionalized $\mathrm{BN}$ nanoplates ranging from $550^{\circ} \mathrm{C}$ to $650^{\circ} \mathrm{C}$. The composites with 50 wt $\%$ functionalized $\mathrm{BN}$ nanoplates had $85 \%$ char residue at $790^{\circ} \mathrm{C}$ compared to $54 \%$ for pure polymer. Composites with higher loadings of functionalized BN nanoplates could retard the main chain scission of the polymer more significantly because their homogeneous distribution as well as the tortuous path in the composites that hindered diffusion of the volatile decomposition products in the composites, compared to that in pure PI. ${ }^{45,46}$ In this way, functionalized BN nanoplates could act as an additive against thermal degradation of the composites as a result of the increase in their concentration.

The dielectric properties of polymeric substrates in flexible circuits play an important role in device performance with regard to electrical signals since both the dielectric constant and dielectric loss significantly influence the signal-carrying capacity and the transmission velocity of signal in electronic systems. The transmission velocity of signal $v$ is inversely proportional to the dielectric constant $e_{r}$ of the flexible substrate, determined by

$$
v=k \frac{c}{\sqrt{\varepsilon_{r}}}
$$

where $\mathrm{C}$ is the velocity of IIght and $\mathrm{K}$ the constant coefficient.

The transmission velocity of signal will decrease when the relative dielectric constant increase. As a result, a low clock rate can be induced by a high dielectric constant for lower signal accumulation, defined as

$$
t_{\mathrm{pd}}=\sqrt{\varepsilon_{r}} \frac{l_{p}}{c}
$$

where $t_{p d}$ is the time of signal propagation delay and $I_{p}$ the transmission distance of the signal.

Increasing the dielectric loss tan d can enlarge the signal attenuation as

$$
\alpha \propto \sqrt{\varepsilon_{r}} f \tan \delta
$$

where $a$ is the signal attenuation and $f$ the working frequency of the device.

Thus, a high dielectric constant could result in a strong negative effect on the signal propagation by increasing the delay time, while a high dielectric loss could induce signal attenuation to form signal distortion. ${ }^{10,47}$

Figure 9 presents the dielectric properties of the PI-based composites filled with functionalized $\mathrm{BN}$ nanoplates, as a function of filler fraction at different frequencies at room temperature. Higher concentrations of functionalized BN nanoplates resulted in higher dielectric constant of composites, as shown in Figure 9(a). At $40 \mathrm{wt} \%$ fraction in the PI-based composite, functionalized BN nanoplates provided a dielectric constant of about 4.4 at $0 \mathrm{~Hz}, 1.3$ more than that of pure PI. BN nanoplates with higher dielectric constant dominated the dielectric constant of the composites as the increment of the concentration. In addition, more minicapacitor networks formed between PI matrix and functionalized BN nanoplates with smaller particle sizes further increased the dielectric constant of composites with higher fractions of functionalized BN nanoplates. ${ }^{48}$ Furthermore, the size of the filler and microstructure of the composites (interface between the filler and matrix) could also influence the final dielectric constant of the polymeric composites with fillers. ${ }^{49}$ There was a higher chance for functionalized BN nanoplates to form random channels with a decrease in the particle size by ball milling, while stronger interfacial interaction between functionalized BN nanoplates and PI matrix could be caused by the silylated functionalization in the composites. A synergistic effect from the decrease in the size of BN nanoplates and silylated functionalization for stronger interfacial interaction led to a lower chance to form voids and pores in composites [inserts of Figure 7(a)]. Thus, good dispersion of $\mathrm{BN}$ nanoplates in the surrounding matrix resulted in the increase in the dipole-dipole interaction between functionalized $\mathrm{BN}$ nanoplates and PI matrix, thereby contributing to a higher dielectric constant. ${ }^{38}$ However, at low frequencies, the composites exhibited much higher dielectric constants than those at high frequencies. The dielectric constant of the composite systems dropped down with the increase in the frequency and was less dependent on the frequency in the measured range from $10 \mathrm{MHz}$ to $30 \mathrm{MHz}$. This was attributed to the leading effect of electronic shift polarization from the composites that could immediately contribute to the formation of the dielectric constant, rather than relaxation polarization that needed more time to influence the dielectric constant with the increased frequency. Early work ${ }^{50}$ reported at low frequencies, the dielectric constant of the composites 

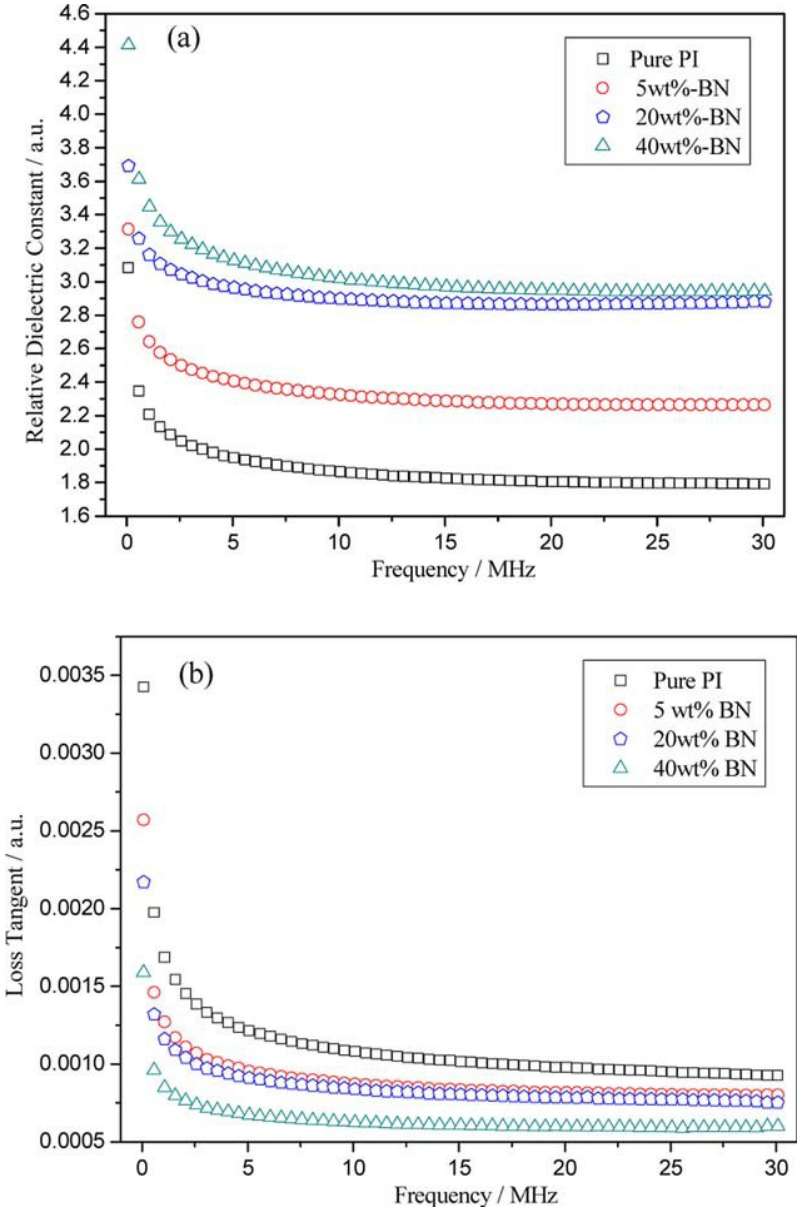

Figure 9. Dielectric properties of pure PI and PI-based composites as a function of the concentrations of functionalized BN nanoplates: dielectric constant (a) and dielectric loss (b). [Color figure can be viewed in the online issue, which is available at wileyonlinelibrary.com.]

strongly depended on the dielectric properties of both the polymer and filler, while at high frequencies, the dielectric constant was sensitive primarily to the filler and its concentration. This indicated that at high frequency the electronic shift polarization of polar groups in polymer chains was too slow to contribute to the dielectric constant of the composite system. Hence, only electronic shift polarization from functionalized BN nanoplates led to the significant decrease in the dielectric constant of the composites with increasing frequency. PI-based composites with functionalized $\mathrm{BN}$ nanoplates could meet the requirement of the dielectric constant for high-frequency circuit even though the dielectric constant reached 4.4 in the condition of $40 \mathrm{wt} \%$ functionalized BN nanoplates.

PI-based composites with functionalized BN nanoplates demonstrated a decreasing tend of the dielectric loss with the increase of filler concentration, as shown in Figure 9(b). This was ascribed to the dominance of BN nanoplates with low dielectric loss. The dielectric loss of the composites significantly decreased at low frequencies but became almost independent on the frequency ranging from $10 \mathrm{MHz}$ to $30 \mathrm{MHz}$, similar to the trend of the dielectric constant. The relationship between the dielectric loss tand and the frequency $\mathrm{x}$ can be defined as

$$
\tan \delta=\frac{\sigma}{\omega \varepsilon_{r}}
$$

where $\mathbf{r}$ is the electric conductıvity of tree charge and $\mathbf{x}$ the frequency in an alternating electric field.

The dielectric loss at a maximum value stemmed from leakage conductance at the frequency of 0 due to no polarized energy consumption. From eq. (4), the dielectric loss will decrease with increasing frequencies since $x_{r} \gg 1$. In addition, the dielectric loss of the dielectric material resulted from relaxation polarization that would consume energy for a steady state of system charges. However, relaxation polarization of polarized composite systems had less time to form the steady state at high frequency. Thus, only electronic shift polarization for no energy consumption dominated the polarized effect of the composite systems, thereby causing a weak dependence of the dielectric loss at high frequency.
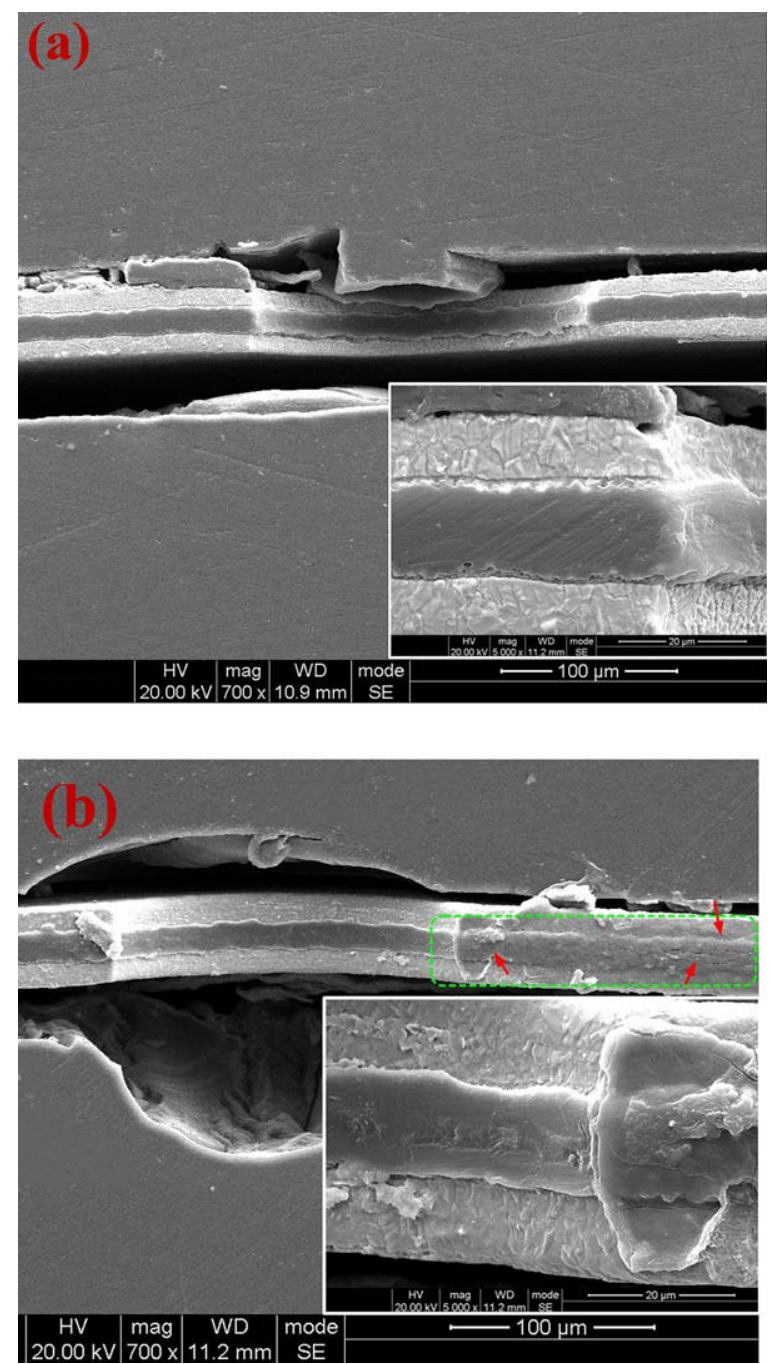

Figure 10. SEM micrographs of functionalized BN nanoplates/PI composites with drilling through holes in a diameter of $200 \mathrm{Im}$ : composite with 30 wt \% functionalized BN nanoplates (a); composite with 40 wt \% functionalized BN nanoplates (b). [Color figure can be viewed in the online issue, which is available at wileyonlinelibrary.com.] 


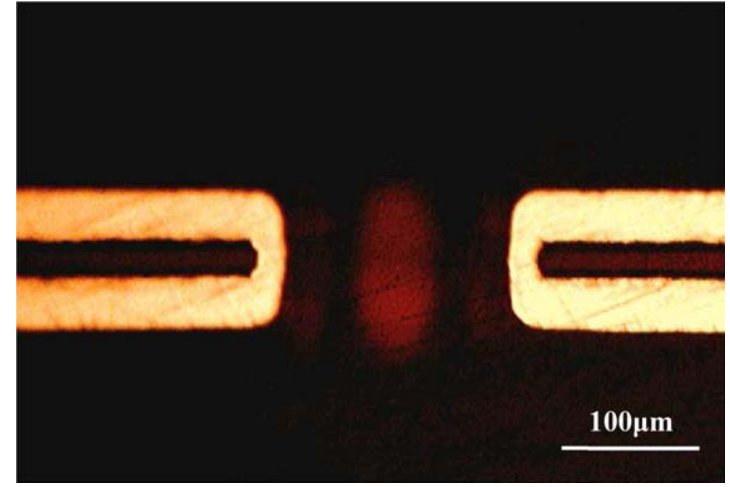

Figure 11. Cross-sectional micrograph of drilling through hole after metallization. [Color figure can be viewed in the online issue, which is available at wileyonlinelibrary.com.]

Application of Functionalized BN Nanoplates/PI Composites Good performance of the polymeric composites could be obtained when increasing the filler concentration in the composites. $^{5,28}$ However, a high filler fraction could dramatically alter the polymer mechanical behavior and challenge the processing of composites for manufacturing flexible circuits. Thus, drilling tests were performed with the developed composites to assess their machinability. Before the drilling tests, functionalized BN nanoplates/PI composites with laminated copper foils on their both sides were attached to the back-up of wood at the bottom and the overlay entry of phenolic resin at the top in order to avoid potential mechanical damage by drilling bits. The effect of filler concentrations on drilling the composites was shown in Figure 10. Cracks around the through hole were found in the PI-based composite with $40 \mathrm{wt} \%$ functionalized BN nanoplates after drilling stress, caused by embrittlement of the composite with high concentration of functionalized BN nanoplates. Such cracks could reduce flexibility of the composites, resulting in reliable problems for flexible circuits. However, PI-based composite with $30 \mathrm{wt} \%$ functionalized BN nanoplates demonstrated good agreement in drilling performance, as shown in Figure 10(a): the through hole exhibited an even annular ring and no cracks. Thus, concentration of nanoplates below $40 \mathrm{wt}$ $\%$ was necessary to achieve the appropriate level of mechanical stability. Figure 11 presents the cross-sectional micrograph of a through hole in the PI-based composite with $30 \mathrm{wt} \%$ functionalized BN nanoplates after copper deposition. It was found that the composite formed satisfying cohesion with copper deposits. Such PI-based composite was in good agreement with high thermal and mechanical properties to meet the requirement of flexible circuits for heat dissipation.

\section{CONCLUSIONS}

In this study, functionalized BN nanoplates with the silylation and smaller particle-size distribution were used to enhance the performance of PI matrix. Thermal conductivity of the composites exhibited a drastic increase as the concentration of functionalized BN nanoplates was raised. Thermal stability of the composites was improved with the increase in concentration of functionalized BN nanoplates. An increase in the dielectric constant and a decrease in the dielectric loss were observed in the
PI-based composites filled with functionalized BN nanoplates when increasing the fractions of functionalized BN nanoplates. At high frequency, both the dielectric constant and the dielectric loss demonstrated low sensitivity to the frequency. Through holes of the composites were in good agreement with the drilling requirement when the concentration of functionalized $\mathrm{BN}$ nanoplates was lower than $40 \mathrm{wt} \%$.

\section{ACKNOWLEDGMENTS}

The authors acknowledge the financial support provided by Key Project of Science and Technology Planning of Guangdong Province China (No. 2012A090300007) and Yuanming Chen also expresses his sincere thanks to the support of China Scholarship Council (No. 201306070032).

\section{REFERENCES}

1. Hin, T. Y.; Liu, C.; Conway, P. P. Surf. Coat. Technol. 2009, 203, 3741.

2. Fjelstad, J. Circuit World 2013, 3, 4.

3. Lu, X. J. Appl. Phys. 2009, 105, 094301.

4. Chen, Y.; He, W.; Zhou, G.; Tao, Z.; Wang, Y.; Luo, D. Circuit World 2013, 39, 133.

5. Henry, A.; Chen, G. Phys. Rev. Lett. 2008, 101, 235502.

6. Hong, J. P.; Yoon, S. W.; Hwang, T.; Oh, J, K.; Hong, S. C.; Lee, Y.; Nam, J. D. Thermochim. Acta 2012, 537, 70.

7. Han, Z.; Fina, A. Progr. Polym. Sci. 2011, 36, 914.

8. Alvarez-Quintana, J.; Martlnez, E.; Pe̋rez-Tijerina, E.; Pe̋rezGarcla, S. A.; RodrIguez-Viejo, J. J. Appl. Phys. 2010, 107, 063713.

9. Dashora, P.; Gupta, G. Polymer 1996, 37, 231.

10. Chen, Y.; He, W.; Zhou, G.; Hu, Y.; Wang, S.; Tao, Z. Polym. Int. 2014, 63, 546.

11. Rossinsky, E.; Mfller-Plathe, F. J. Chem. Phys. 2009, 130, 134905.

12. Zhou, T.; Wang, X.; Liu, X.; Xiong, D. Carbon 2010, 48, 1171.

13. Ganguli, S.; Roy, A. K.; Anderson, D. P. Carbon 2008, 46, 806.

14. Yu, A.; Ramesh, P.; Sun, X.; Bekyarova, E.; Itkis, M. E.; Haddon, R. C. Adv. Mater. 2008, 20, 4740.

15. Hung, M. T.; Choi, O.; Ju, Y. S.; Hahn, H. T. Appl. Phys. Lett. 2006, 89, 023117.

16. Zhi, C.; Bando, Y.; Tang, C.; Kuwahara, H.; Golberg, D. Adv. Mater. 2009, 21, 2889.

17. Li, S.; Qi, S.; Liu, N.; Cao, P. Thermochim. Acta 2011, 523, 111.

18. Ng, H. Y.; Lu, X.; Lau, S. K. Polym. Compos. 2005, 26, 778.

19. Wang, J.; Yi, X. J. Appl. Polym. Sci. 2003, 89, 3913.

20. Wang, J. Y.; Yang, S. Y.; Huang, Y. L.; Tien, H. W.; Chin, W. K.; Ma, C. C. M. J. Mater. Chem. 2011, 21, 13569.

21. Jiajun, W.; Xiao-Su, Y. Compos. Sci. Technol. 2004, 64, 1623. 
22. Ling, W.; Gu, A.; Liang, G.; Yuan, L. Polym. Compos. 2010, $31,307$.

23. Li, T. L.; Hsu, S. L. C. J. Phys. Chem. B. 2010, 114, 6825.

24. Li, C. H.; Peterson, G. P. J. Appl. Phys. 2006, 99, 084314.

25. Teng, C. C.; Ma, C. C. M.; Lu, C. H.; Yang, S. Y.; Lee, S. H.; Hsiao, M. C.; Yen, M. Y.; Chiou, K. C.; Lee, T. M. Carbon 2011, 49, 5107.

26. Fang, M.; Zhang, Z.; Li, J.; Zhang, H.; Lu, H.; Yang, Y. J. Mater. Chem. 2010, 20, 9635.

27. Yang, K.; Gu, M. Compos. A 2010, 41, 215.

28. Monticelli, O.; Calabrese, M.; Gardella, L.; Fina, A.; Gioffredi, E. Eur. Polym. J. 2014, 58, 69.

29. Kuo, S. W.; Chang, F. C. Prog. Polym. Sci. 2011, 36, 1649.

30. Xie, Y.; Hill, C. A.; Xiao, Z.; Militz, H.; Mai, C. S. Compos. A 2010, 41, 806.

31. Sainsbury, T.; Ikuno, T.; Okawa, D.; Pacile, D.; Frechet, J. M.; Zettl, A. J. Phys. Chem. C. 2007, 111, 12992.

32. Jo, B. W.; Kim, C. H.; Tae, G. H.; Park, J. B. Constr. Build. Mater. 2007, 21, 1351.

33. Lee, M.; Khan, S. B.; Akhtar, K.; Han, H.; Seo, J. Int. J. Electrochem. Sci. 2013, 8, 4225.

34. Ramanathan, T.; Abdala, A. A.; Stankovich, S.; Dikin, D. A.; Herrera-Alonso, M.; Piner, R. D.; Adamson, D. H.; Schniepp, H. C.; Chen, X.; Ruoff, R. S.; Nguyen, S. T.; Aksay, I. A.; Prud'Homme, R. K.; Brinson, L. C. Nat. Nanotechnol. 2008, 3, 327.

35. Ghosh, S.; Bao, W.; Nika, D. L.; Subrina, S.; Pokatilov, E. P.; Lau, C. N.; Balandin, A. A. Nat. Mater. 2010, 9, 555.

36. Liu, L.; Chen, X. J. Appl. Phys. 2010, 107, 033501.
37. Martin, P.; Aksamija, Z.; Pop, E.; Ravaioli, U. Phys. Rev. Lett. 2009, 102, 125503.

38. Dang, Z. M.; Yu, Y. F.; Xu, H. P.; Bai, J. Compos. Sci. Technol. 2008, 68, 171.

39. Zhou, T.; Zha, J. W.; Cui, R. Y.; Fan, B. H.; Yuan, J. K.; Dang, Z. M. ACS Appl. Mater. Interfaces 2011, 3, 2184.

40. Gu, J. W.; Zhang, Q.; Zhang, J.; Wang, W. Polym. Plast. Technol. Eng. 2010, 49, 1385.

41. Ling, W.; Gu, A.; Liang, G.; Yuan, L.; Liu, J. Polym. Adv. Technol. 2010, 21, 365.

42. Garg, J.; Poudel, B.; Chiesa, M.; Gordon, J. B.; Ma, J. J.; Wang, J. B.; Ren, Z. F.; Kang, Y. T.; Ohtani, H.; Nanda, J.; McKinley, G. H.; Chen, G. J. Appl. Phys. 2008, 103, 074301.

43. Evans, W.; Prasher, R.; Fish, J.; Meakin, P.; Phelan, P.; Keblinski, P. Int. J. Heat Mass Tran. 2008, 51, 1431.

44. Peters, J. E.; Papavassiliou, D. V.; Grady, B. P. Macromolecules 2008, 41, 7274.

45. Kim, H. S.; Park, B. H.; Choi, J. H.; Yoon, J. S. J. Appl. Polym. Sci. 2008, 109, 3087.

46. Liufu, S. C.; Xiao, H. N.; Li, Y. P. Polym. Degrad. Stabil. 2005, 87, 103.

47. Lanagan, M. T.; Yamamoto, J. K.; Bhalla, A.; Sankar, S. G. Mater. Lett. 1989, 7, 437.

48. Li, Y. J.; Xu, M.; Feng, J. Q.; Dang, Z. M. Appl. Phys. Lett. 2006, 89, 072902.

49. Ozmusul, M. S.; Picu, R. C. Polym. Composite. 2002, 23, 110.

50. Popielarz, R.; Chiang, C. K.; Nozaki, R.; Obrzut, J. Macromolecules 2001, 34, 5910. 